\title{
Mitbestimmung des Personals beim Wechsel der Vorsorgeeinrichtung
}

\section{Kommentar zu BGE 146 V 169}

Die Kündigung des Anschlussvertrags mit der Vorsorgeeinrichtung durch den Arbeitgeber setzt die vorgängige Zustimmung des Personals voraus. Es handelt sich dabei um ein echtes Mitbestimmungsrecht der Arbeitnehmenden. Die Zustimmung muss ausdrücklich und vorgängig erteilt werden. Fehlt sie, ist die Kündigung ungültig und entfaltet keine Wirkung.

I. Einführung

II. Sachverhalt

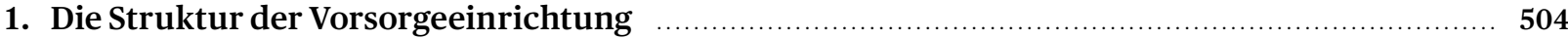

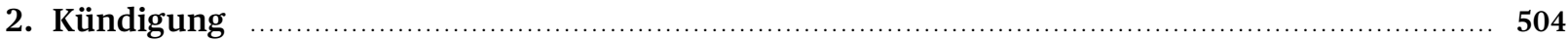

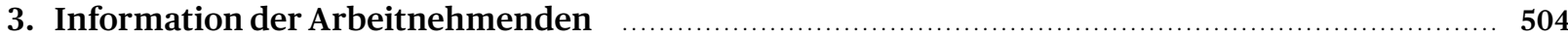

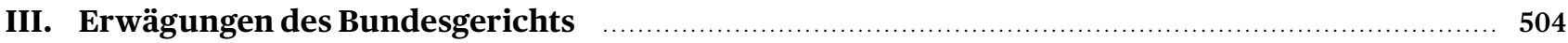

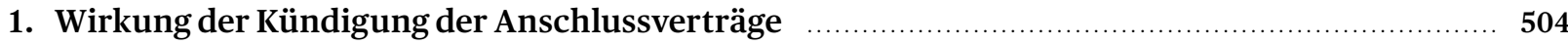

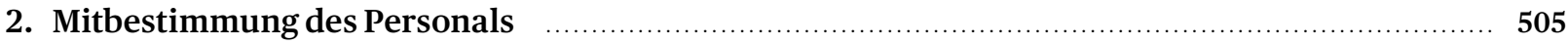

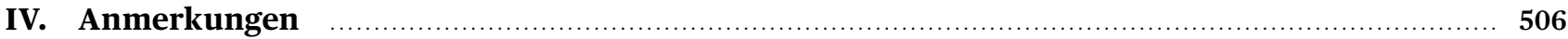

Zitiervorschlag:

MARC HÜRZELER / RAFFAELLA BIAGGI,

Mitbestimmung des Personals beim Wechsel der

Vorsorgeeinrichtung, sui generis 2020, S. 503

Prof. Dr. Marc Hürzeler, Sozialversicherungsfachmann mit eidg.FA, Ordinarius für Sozialversicherungsrecht an der Universität Luzern, Geschäftsführer der Prof. Dr. Marc Hürzeler GmbH, Küttigen (mh@ marc-huerzeler.ch). Lic. iur. Raffaella Biaggi, Advokatin, Fachanwältin SAV Haftpflicht- und Versicherungsrecht, Basel(rb@advokaturbiaggi.ch).

URL: sui-generis.ch/161

DOI: https://doi.org/10.21257/sg.161

Dieses Werk ist lizenziert unter einer Creative Commons Namensnennung - Weitergabe unter gleichen Bedingungen 4.0 International 


\section{Einführung}

1 Bundesverwaltungsgericht (unter der Referenz A-3248/ 2018) und Bundesgericht ${ }^{1}$ setzen sich im vorliegenden Fall mit formellen Fragen zur gerichtlichen Zuständigkeit bei Teilliquidation nach Art. $53 d \mathrm{Abs} .6 \mathrm{BVG}^{2}$ auseinander und grenzen die Verfahrenswege - Klageverfahren gemäss Art. 73 BVG einerseits, Beschwerdeverfahren nach Art. 74 BVG anderseits - gegeneinander ab. Sie setzen sich zudem mit der Frage der Voraussetzungen einer Teilliquidation bei einer zweistufig organisierten Vorsorgeeinrichtung auseinander und beleuchten und klären schliesslich die Frage, unter welchen Voraussetzungen dem echten Mitwirkungsrecht der Arbeitnehmenden beim Wechsel der Vorsorgeeinrichtung Genüge getan ist. Zudem äussert sich das Bundesgericht dazu, wer für die Prüfung der Gültigkeit der Kündigung verantwortlich ist.

\section{Sachverhalt}

\section{Die Struktur der Vorsorgeeinrichtung}

2 Die vor Bundesgericht beschwerdeführende Vorsorgeeinrichtung ist eine Sammelstiftung, die allen dem Schweizerischen Gewerbeverband angehörigen Organisationen die Durchführung der beruflichen Vorsorge anbietet. Diese Organisationen (vorliegend die Verbände) schliessen sich mittels Anschlussvertrages bei der Sammelstiftung an und bilden je ein Vorsorgewerk. Die angeschlossenen Vorsorgewerke werden hinsichtlich Buchhaltung und Reglementen getrennt geführt und es besteht keine Solidarität zwischen den Vorsorgewerken (Struktur einer Sammelstiftung). Die einer Organisation angehörigen Arbeitgeber treten wiederum dem Vorsorgewerk bei und schliessen zu diesem Zweck eine Beitrittsvereinbarung ab. Buchhaltung und Reglemente werden auf dieser Ebene nicht vollständig getrennt geführt. Es bestehen Solidaritäten zwischen den Arbeitgebern im Rahmen des gemeinsamen Vorsorgewerkes (Struktur analog einer Gemeinschaftsstiftung).

\section{Kündigung}

3 Die Verbände kündigten die Anschlussverträge mit der Sammelstiftung und verlangten die Durchführung einer Teilliquidation. Die Kündigungen wurden durch die Sammelstiftung per 31. Dezember 2017 vorbehaltlos bestätigt und ausdrücklich als gültig bezeichnet. Zudem kündigte die Sammelstiftung in der Folge den Verbandsversicherungsvertrag, den sie für die Pensionskasse mit drei Versicherungsgesellschaften abgeschlossen hatte, ebenfalls per 31. Dezember 2017.

1 BGE 146 V 169.

2 Bundesgesetz über die berufliche Alters-, Hinterlassenen- und Invalidenvorsorge vom 25. Juni 1982 (BVG; SR 831.40).
Im Gerichtsverfahren verneinte die Sammelstiftung das 4 Vorliegen eines Teilliquidationstatbestandes, weil nur die Anschlussverträge gekündigt worden seien, nicht aber die einzelnen Beitrittsvereinbarungen aller Arbeitgeber zu den auf der Grundlage der Anschlussverträge entstandenen Vorsorgewerken. Zudem machte die Sammelstiftung geltend, dass die Mitwirkungsrechte der einzelnen Arbeitnehmer nicht gewahrt worden seien, was zur Nichtigkeit der Kündigungen führe.

\section{Information der Arbeitnehmenden}

Die Arbeitgeber waren über den Beschluss der Gründer- 5 bzw. Trägerverbände informiert worden, die Anschlussverträge bei der Sammelstiftung zu kündigen. Mit einem späteren Schreiben wurden die Arbeitgeber darüber informiert, dass die Pensionskasse neu autonom geführt und kollektiv in eine neue Stiftung überführt werden solle. Die Arbeitgeber wurden aufgefordert, den Mitarbeitenden ein Beiblatt mit Informationen zum Pensionskassenwechsel umgehend abzugeben. Den Arbeitgebern wurden drei Möglichkeiten der Willensäusserung eingeräumt: 1) Unterzeichnung und Retournierung der Vereinbarung mit Einverständnis der Mehrheit der Mitarbeitenden, 2) Mitteilung über die Ablehnung des Wechsels oder 3) bei Ausbleiben einer Reaktion werde das Einverständnis und damit das stillschweigende Zustandekommen der Vereinbarung angenommen. Zwei Arbeitgeber machten von der Möglichkeit Gebrauch, die Ablehnung der Überführung kundzutun. Die übrigen Arbeitgeber liessen sich nicht vernehmen. Aufsichtsbehörde und Bundesverwaltungsgericht gingen aufgrund dieses Sachverhalts davon aus, dass dem Mitbestimmungsrecht des Personals Genüge getan worden war und ein Teilliquidationstatbestand vorliege.

\section{Erwägungen des Bundesgerichts}

\section{Wirkung der Kündigung der Anschlussverträge}

Zur Struktur der Stiftung stellte das Bundesgericht auf der 6 Grundlage der Erwägungen des Bundesverwaltungsgerichts fest, dass sich aus Stiftungsurkunde und Teilliquidationsreglement ergebe, dass zwischen der Stiftung einerseits und den drei Gründerverbänden des Vorsorgewerks andererseits Anschlussvereinbarungen abgeschlossen worden waren. Die Beitrittsvereinbarungen hingegen wurden zwischen der Pensionskasse als Teil der Stiftung und dem einzelnen Arbeitgeber geschlossen. Weder in der Anschlussvereinbarung selbst noch im Teilliquidationsreglement ist festgelegt, dass im Falle der Kündigung der Anschlussvereinbarung zusätzlich auch alle Beitrittsvereinbarungen der einzelnen, an das Vorsorgewerk angeschlossenen Arbeitgeber aufgelöst werden müssen, damit ein Austritt möglich wäre (E.3.1.2). 
7 Das Bundesgericht kritisiert die Auffassung der Vorinstanz, wonach bereits die Kündigung der Anschlussverträge durch die Gründerverbände die Teilliquidation der Stiftung auslöse und dass entsprechend die Beitrittsvereinbarungen keine eigenständige Bedeutung hätten und daher nicht zusätzlich gekündigt werden müssten (E. 3.2.1).

8 Das Bundesgericht hält fest, dass der materiell-rechtliche Anschluss nach Art.11 Abs. 1 BVG in concreto durch die Beitrittsvereinbarungen zwischen den Pensionskassen und den einzelnen Arbeitgebern erfolge, wobei es keine Rolle spiele, dass der einzelne Arbeitgeber aufgrund der ihn bindenden GAV-Vorschriften allenfalls keine Wahl habe, sich einer anderen als der Verbandspensionskasse anzuschliessen. Ebenfalls als irrelevant bezeichnet das Bundesgericht die Tatsache, dass das Vorsorgewerk über keine Rechtspersönlichkeit verfügt. Die Kündigung der Anschlussvereinbarung zielt nach den Feststellungen des Bundesgerichts auf eine Änderung des betrieblichen Umfelds ab und begründet für sich allein noch keinen Abgang von Versicherten (E.3.2.2).

9 Weiter hält das Bundesgericht fest, dass jeder Teilliquidationstatbestand zwingend mit dem Austritt aus der Vorsorgeeinrichtung verbunden ist. Zwar schreiben weder Anschlussvereinbarung noch Teilliquidationsreglement vor, dass im Falle der Kündigung der Anschlussvereinbarung durch die Trägerverbände zusätzlich alle Beitrittsvereinbarungen aufzulösen sind. Umgekehrt wird aber in der Beitrittsvereinbarung die Auflösung des Anschlussvertrages auch nicht als Kündigung der Beitrittsvereinbarungen anerkannt (E.3.2.3).

10 Gemäss den Ausführungen des Bundesgerichts stehen weder die Aufsichtsbehörden noch die AHV-Ausgleichskassen oder die Auffangeinrichtung BVG in der Pflicht, den ordnungsgemässen Verlauf des Pensionskassenwechsels zu kontrollieren. Vielmehr ist es Sache der abgebenden Pensionskasse, zu prüfen, ob das Verfahren korrekt eingehalten wurde (E. 3.2.3.1).

11 Das Bundesgericht lässt aber offen, ob die Auffassung der Vorinstanz, wonach mit der Kündigung der Anschlussverträge auch die Rechtsverhältnisse der einzelnen Arbeitgeber mit der Pensionskasse aufgelöst sind, richtig oder falsch ist. Im konkreten Fall wurde die Kündigung der Anschlussvereinbarungen ausdrücklich und ohne Vorbehalt durch die Stiftung bestätigt. Diese Bestätigung bezog sich nach Auffassung des Bundesgerichts nicht nur auf den Erhalt der Kündigung, vielmehr wurde ihre Gültigkeit ausdrücklich festgehalten. Weiter löste die Stiftung ihrerseits den Verbandsversicherungsvertrag mit drei Versicherungsgesellschaften, die sie betreffend Pensionskasse abgeschlossen hatte, ebenfalls auf. Damit erlosch der Versi- cherungsschutz für sämtliche Versicherte und die Stiftung brachte ihr Desinteresse an der Pensionskasse zum Ausdruck. Obwohl sie einräumte, darüber orientiert worden zu sein, dass keine Konsultation des Personals der angeschlossenen Betriebe vorausgegangen war, hat sie den Verbandsversicherungsvertrag aufgelöst (E.3.2.3.1). Wenn sie sich nun darauf berufen möchte, dass eine formelle Kündigung der Arbeitgeber der Beitrittsvereinbarungen notwendig gewesen wäre, verhalte sie sich widersprüchlich und verdiene darin keinen Schutz (E.3.2.3.2).

\section{Mitbestimmung des Personals}

Für das Bundesgericht steht verbindlich fest, dass die 12 fragliche Kündigung ohne Einverständnis des davon betroffenen Personals ausgesprochen und dieses erst nachträglich in Kenntnis gesetzt worden ist (E.4.4).

Nach Art. 11 Abs. $3^{\text {bis }}$ BVG kann ein bestehender An- 13 schluss an eine Vorsorgeeinrichtung und der Wiederanschluss an eine neue Vorsorgeeinrichtung durch den Arbeitgeber nur im Einverständnis mit dem Personal oder der allfälligen Arbeitnehmervertretung erfolgen. Letztere ist nicht mit der paritätischen Verwaltung einer Vorsorgeeinrichtung zu verwechseln. Vorausgesetzt ist das Einverständnis des Personals und somit der Arbeitnehmenden (E. 4.1.1, E. 4.3.2.1). Das Bundesgericht stellt fest, dass die Stiftung nicht unter den Schutz des Mitwirkungsgesetzes ${ }^{3}$ (Art. 10 lit. d) falle, nachdem diese Mitwirkungsrechte den Arbeitnehmervertretungen zustehen und auch diese klageberechtigt sind (Art.15 Abs. 1 Mitwirkungsgesetz) (E. 4.1.2).

Entgegen der Auffassung des Bundesverwaltungsge- 14 richts geht das Bundesgericht davon aus, dass die Annahme einer stillschweigenden Übereinkunft den Anforderungen von Art. 11 Abs. $3^{\text {bis }}$ BVG nicht genügt. Es handle sich bei Art. 11 Abs. $3^{\text {bis }}$ BVG nicht um eine blosse Ordnungsvorschrift (E. 4.4.). Das Wort «Einverständnis» erfordere deutlich die diesbezügliche Einwilligung des Personals und schreibe diesem eine aktive Rolle zu. Ein Einverständnis erfolge zudem im Voraus (E. 4.3.1). Es muss für alle offen und transparent sein, welcher Einrichtung sich der Arbeitgeber zu welchen Konditionen anschliessen will (E. 4.3.2.1). Das Bundesgericht präzisiert zudem, dass dem Gesetzgeber eine gemeinsame Entscheidung von Arbeitgeber und Arbeitnehmern vorschwebte und er dem Personal ein besonderes, vorsorgespezifisches Mitwirkungsrecht einräumen wollte. Es bedürfe der Zustimmung des Personals zum Anschlusswechsel. Dieser Akt sei als kollektive Grösse zu begreifen und nicht als eine Kumulation individueller Rechte, die

3 Bundesgesetz über die Information und Mitsprache der Arbeitnehmerinnen und Arbeitnehmer in den Betrieben vom 17. Dezember 1993 (Mitwirkungsgesetz; SR 822.14). 
jedem einzelnen Arbeitnehmer zustehe (E. 4.3.2.2). Die Einigung sei konstitutiv. Daraus erhelle, dass es bei Uneinigkeit keine gestaffelte Beschlussfassung im Sinne einer «nachgeordneten» Genehmigung gebe. Dies könne bei einer «Konsens-Kündigung» i.S.v. Art.11 Abs. $3^{\text {bis BVG }}$ nicht anders sein (E. 4.3.3.1). Aufgrund ihrer vertraglichen Natur stellt die anschlussvertragliche Kündigung ein einseitiges Gestaltungsrecht, das grundsätzlich bedingungsfeindlich und unwiderruflich ist, dar. Entsprechend kann die Kündigung ohne die notwendige Zustimmung des Personals keine Wirkung entfalten (E. 4.3.3.2).

15 Als unzulässig versteht es das Bundesgericht, das Mitwirkungsrecht in ein reines Oppositionsrecht umzuwandeln. Dies würde eine Schwächung des Mitwirkungsrechts bedeuten, da nicht gemeinsam die Gründe abgewogen und bereinigt würden, sondern die Arbeitnehmer vor vollendete Tatsachen gestellt würden, sie nur entweder annehmen oder ablehnen könnten. Der Entscheid über die Kündigung kann aufgrund des kollektiven Charakters des Mitwirkungsrechts nicht einzelnen Arbeitnehmenden überlassen werden (E. 4.3.3.3).

16 Auch Praktikabilitätsgründe rechtfertigten schliesslich die Beschneidung der Mitbestimmung der Arbeitnehmenden nicht. Die Information muss vorgängig und umfassend sein. Die nötige Vorlaufzeit sei kalkulierbar. Einen Unterschied zwischen autonomen Stiftungen und Sammelstiftungen gebe es nicht (E. 4.3.4). Die Kündigung ist damit ungültig und ohne Rechtsfolge (E. 4.4).

\section{Anmerkungen}

17 Das zitierte Urteil des Bundesgerichts bringt in vielerlei Hinsicht Konkretisierungen mit sich.

Zum einen hält das Bundesgericht fest, dass die bisherige Vorsorgeeinrichtung zu prüfen hat, ob die Voraussetzungen für eine gültige Kündigung des Anschlussverhältnisses erfüllt sind. Dazu gehört auch die aktive Mitbestimmung des Personals, die durch die kündigende Partei entsprechend detailliert dargelegt werden muss. Je nachdem ob die Vorsorgeeinrichtung ein Interesse am Verbleib oder am Abgang der Versicherten hat, wird sie diese Frage grosszügiger oder strenger beurteilen.

Zum anderen geht das Bundesgericht von einer aktiven Mitbestimmung des Personals aus, die sich nicht darin erschöpft, ja oder nein zu einer vorgeschlagenen Lösung zu sagen. Vielmehr wird ein gemeinsamer Prozess von Arbeitgeber und Arbeitnehmervertretung bzw. - wo keine solche existiert - Arbeitnehmerschaft bei der Frage nach dem Wechsel, der Auswahl der Vorsorgeeinrichtung und den Modalitäten als vom Gesetzgeber gewollt angesehen.
Die Zustimmung der Arbeitnehmerschaft zum Wechsel 20 der Vorsorgeeinrichtung muss vorgängig erteilt werden. Damit die Gültigkeit der Kündigung bestätigt werden kann, muss grundsätzlich der gesamte Prozess mit sämtlichen geflossenen Informationen dokumentiert werden.

Das Bundesgericht erachtet gerade Praktikabilitäts- 21 gründe als nicht geeignet, von diesem vom Gesetzgeber gewollten vorsorgespezifischen Mitbestimmungsrecht abzuweichen, und setzt dies der nicht zulässigen, nachträglich und konkludent einzuholenden Zustimmung entgegen. Künftig werden damit beabsichtigte Wechsel der Vorsorgeeinrichtung gut dokumentiert werden müssen, das Personal ist über die objektiv relevanten Aspekte zu informieren und hat ausdrücklich zuzustimmen, um einen Wechsel überhaupt zu ermöglichen.

Trotz der vielen Klärungen zum Mitbestimmungsrecht 22 nach Art. 11 Abs. 3 bis BVG im vorliegenden Urteil bleiben darüber hinaus weiterhin offene Fragen. Nicht klären musste das Bundesgericht namentlich den Anwendungsbereich des Mitbestimmungsrechts: Gilt dieses nur im Bereich der obligatorischen beruflichen Vorsorge oder sind auch Anschlussverhältnisse der weitergehenden und ausserobligatorischen beruflichen Vorsorge erfasst? Während die Gesetzessystematik - Art. 11 Abs. $3^{\text {bis }}$ BVG findet sich weder im Katalog von Art. 49 Abs. 2 BVG noch in denjenigen von Art. $89 a$ Abs. 6 und $7 \mathrm{ZGB}^{4}$ - für einen auf die obligatorische berufliche Vorsorge beschränkten Wirkungskreis hindeutet, wäre nach dem Sinn und Zweck der Norm eher von einer umfassenden Anwendung auszugehen, da sich die Vorsorgepläne gerade in der weitergehenden beruflichen Vorsorge deutlich unterscheiden können. De facto bleibt diese Differenzierung zumindest bei umhüllenden Vorsorgelösungen letztlich weniger bedeutsam, da hier obligatorische und weitergehende berufliche Vorsorge auf einem einzigen Anschlussverhältnis beruhen. Schliesslich bleibt der Begriff des mitbestimmungsberechtigten «Personals», wie ihn Art. 11 Abs. 3 bis BVG nicht klar definiert. Gemäss dem vorliegenden Urteil fallen grundsätzlich alle Arbeitnehmerinnen und Arbeitnehmer des betreffenden Arbeitgebers darunter. Tatsächlich dürften es aber nur diejenigen sein, die auch der Vorsorgeeinrichtung als versicherte Personen angehören, da nur diesen mit Blick auf einen Wechsel der Vorsorgeeinrichtung erhebliche und besonders schützenswerte Interessen beizumessen sind.

4 Schweizerisches Zivilgesetzbuch vom 10. Dezember 1907 (ZGB SR 210). 